\title{
BMJ Open Protocol for a prospective observational study: the Australia and New Zealand Diabetic and Ischaemic Foot Outcomes Study (ANZ-DIFOS)
}

\author{
Odette Hart (D) , ${ }^{1,2}$ Shirley Jansen (D) , $3,4,5$ Robert Fitridge (1) , 6,7 \\ Manar Khashram (1) ${ }^{1,2}$
}

To cite: Hart 0, Jansen S, Fitridge $\mathrm{R}$, et al. Protocol for a prospective observational study: the Australia and New Zealand Diabetic and Ischaemic Foot Outcomes Study (ANZ-DIFOS). BMJ Open 2021;11:e050833. doi:10.1136/ bmjopen-2021-050833

- Prepublication history and additional supplemental material for this paper are available online. To view these files, please visit the journal online (http://dx.doi.org/10.1136/ bmjopen-2021-050833)

Received 03 March 2021 Accepted 19 August 2021

Check for updates

(C) Author(s) (or their employer(s)) 2021. Re-use permitted under CC BY-NC. No commercial re-use. See rights and permissions. Published by BMJ.

For numbered affiliations see end of article.

Correspondence to

Dr Odette Hart;

ohart.research@gmail.com

\section{ABSTRACT}

Introduction Diabetic foot disease is a common condition globally and is over-represented in indigenous populations. The propensity for patients with diabetic foot disease to undergo minor or major limb amputation is a concern. Diabetic foot disease and lower limb amputation are debilitating for patients and have a substantial financial impact on health services. The purpose of this multicentre study is to prospectively report the presentation, management and outcomes of diabetic foot disease, to validate existing scoring systems and assess long term outcomes for these patients particularly in relation to major limb amputation.

Methods and analysis This is a multisite, international, prospective observational study, being undertaken at Waikato Hospital, New Zealand (NZ); Sir Charles Gairdner Hospital, the Royal Adelaide Hospital and the Queen Elizabeth Hospital, Australia. Consecutive participants with diabetic foot disease that meet inclusion criteria and agree to participate will be recruited from multidisciplinary team diabetic foot clinic, vascular clinic, dialysis and admission to hospital. Follow-up of participants will occur at 1, 3, 6 and 12 months. At recruitment and follow-up reviews, information about service details, demographic and clinical history, wound data and discharge information will be recorded. The primary outcomes are the time to wound healing, major amputation, overall mortality and amputation-free survival at 12 months. This study started in NZ in August 2020 and will commence in Australian sites in early 2021.

Ethics and dissemination New Zealand Central Health and Disability Ethics Committee (20/CEN/122), Waikato DHB Research Department (RD0020044), Quality Improvement HoD Sir Charles Gairdner Hospital (39715) and the Central Adelaide Local Health Network (CALHN) Human Research Ethics Committee (13928). Results will be presented at international conferences and published in peer-reviewed journals.

Trial registration number Australian New Zealand Clinical Trials Registry (ACTRN12621000337875).

\section{INTRODUCTION}

\section{Diabetic foot disease}

Diabetic foot disease is a frequent and costly complication of diabetes which requires
Strengths and limitations of this study

- The Australia and New Zealand Diabetic and Ishaemic Foot Outcomes Study (ANZ-DIFOS) will provide evidence of the presentation, management and outcomes of diabetic foot disease from an international collaboration.

- The collaborative recruitment across multiple hospital sites will allow entry of a greater number of participants into the study.

- The prospective study design will allow participants to be followed over time, and thus wound and arterial anatomy scoring systems can be assessed for their predictability of major limb amputation and mortality at 1 year.

- The primary limitations of this study are the potential for missing data points on recruitment or follow-up assessment, and loss of patients to follow-up.

complex multidisciplinary management in the healthcare setting. Diabetic foot disease is defined by the International Working Group on the Diabetic Foot (IWGDF) guidelines as infection, ulceration or destruction of tissues of the foot associated with neuropathy and/or peripheral artery disease in the lower extremity of a person with (a history of) diabetes mellitus'. ${ }^{1}$ One in four people with diabetes will develop a diabetes-related foot ulcer during their lifetime, ${ }^{2}$ and the risk of recurrent ulceration after healing in 1 year is $40 \%{ }^{3}$ This is despite tertiary level multidisciplinary team (MDT) efforts in diabetic foot disease treatment and ulcer prevention.

\section{Lower limb amputation}

The propensity for diabetic foot disease to lead to minor or major limb amputation is a significant compounding issue, despite highrisk multidisciplinary foot clinics, publicly available orthotic care and tertiary vascular surgical units. Major limb amputation is defined as amputation above the ankle, and 
minor amputation as that below the ankle. ${ }^{4}$ Major limb amputation does not only carry psychological trauma and a lower quality of life, but also has been shown to be associated with a high 30-day and midterm mortality. ${ }^{45}$

\section{Burden of disease}

Managing diabetic foot disease may account for up to $20 \%$ of all spending on diabetes mellitus. ${ }^{5}$ These include costs for treating ulcers, gangrene and infection; surgical costs for debridement, revascularisation and amputations; and loss of patient productivity and quality of life. In Australia, the burden of diabetic foot disease is estimated to cost $\$ A 1.6$ billion year. ${ }^{6}$ Similarly, in New Zealand (NZ), it has been estimated that the average cost of managing a diabetic foot ulcer is approximately $\$ 30000$ NZD per wound. ${ }^{7}$

The burden of diabetic foot disease is compounded by the fact that the prevalence of diabetes is over-represented in the indigenous population in both $\mathrm{NZ}$ and Australia. A systematic review has shown that Aboriginal and Torres Strait Islander Australians are 3-6 times more likely to experience diabetic-foot-related complications compared with non-indigenous Australians ${ }^{8}$ and outcome data are similar in NZ Māori people. ${ }^{9}$ Māori patients with diabetes are $65 \%$ more likely to require an above knee amputation compared with diabetic European New Zealanders (adjusted HR 1.65, 95\% CI 1.37 to 1.97). ${ }^{4}$ Knowledge regarding modes of presentation, referral patterns and access to diabetic foot clinics for indigenous populations might help to understand the reasons behind this inequality and develop systematic processes to support initiatives to improve outcomes.

\section{Current challenges and evidence gap}

In 2019, the IWGDF guidelines identified eight factors that were meaningfully related to the diabetic foot ulcer outcomes of healing, hospitalisation, amputation and mortality. ${ }^{10}$ These are the presence of end stage renal disease, peripheral artery disease, loss of protective sensation in the limb and the ulcer factors of area, depth, location (forefoot/hindfoot), number (single/multiple) and infection. ${ }^{10}$ These factors are yet to be reviewed in large scale research across the Australian and NZ populations.

Additionally, in an attempt to standardise reporting of diabetic foot ulcers in clinical practice and research, the Society for Vascular Surgery Lower Extremity Guidelines Committee created the Lower Extremity Threatened Limb WIfI Classification System. ${ }^{11}$ The WIfI classification stratifies disease burden from a lower limb wound in peripheral vascular disease or diabetic foot disease, based on the description of the Wound characteristics, the measurement of Ischaemia at the foot and the degree of Foot Infection. ${ }^{11}$ The WIfI classification can be used to predict major limb amputation at 1 year and benefit of revascularisation. To date, there has been inconsistency in reporting of wound grading, unstandardised vascular assessments and retrospective validation of the WIfI score. This is despite the fact that the recent global vascular guidelines on the management of chronic limbthreatening ischaemia have endorsed the use of the WIfI staging system and have also introduced a new global anatomic staging system. ${ }^{12}$

Impaired arterial perfusion to the foot is a prominent factor to the development of foot ulceration in diabetic foot disease, hence, the accurate assessment of foot perfusion is an essential step in patient management. ${ }^{13}$ The standard approaches to imaging of the lower limb vascular system include ultrasound, CT with contrast and MRI with contrast. These have limitations including expense, access to service within the public health sector, risk of contrast-induced nephropathy due to contrast agent administration and radiation exposure. While ultrasound of the lower limb vasculature removes some of these risks, it however does not routinely measure perfusion within the foot vessels. Hence, new techniques for assessing lower limb and foot perfusion are required.

Pedal acceleration time (PAT) is a novel measure of foot perfusion using duplex ultrasound and provides a precise time measurement in milliseconds as measured from the start of the systolic up-rise to the peak of systole at the mid-artery. ${ }^{14}$ The theory behind PAT is that severity of vascular disease can be quantified based on how fast the red blood cells are travelling within the blood vessel that supplies the affected angiosome depending on wound location. ${ }^{14}$ PAT measurements are grouped into four classes of severity which correlate with ankle-brachial index (ABI) and clinical presentation $(\mathrm{p}<0.001){ }^{14}$ Overall, a normal PAT is defined as $<120 \mathrm{~ms}$, whereas a severe PAT $>225 \mathrm{~ms}$, however, current published research has excluded diabetic participants. ${ }^{14}$

This collaborative project between Australia and NZ will create a prospective database that captures a vast amount of data in diabetic foot disease, including the WIfI score, factors related to patient outcomes and will implement PAT. This collaborative approach builds a strong clinical research network and harnesses the power of a multinational and multicentre study. This is in line with recommendations from the national body representing diabetic foot disease- Diabetes Feet Australia. Diabetes Feet Australia has recommended that clinical trial networks focused on diabetes-related foot disease need to be developed in an attempt to guide national policies and provide leadership. ${ }^{6}$ Moreover, this type of multicentre study is also advocated for by The New Zealand Health Research Prioritisation Framework (2019) as required to improve treatments and hasten health solutions for New Zealanders. ${ }^{15}$ Additionally, the IWGDF has prioritised 10 important future studies in this field, suggesting as particularly useful research topics the assessment, natural history and management strategies of the diabetic foot with the presence of peripheral arterial disease. ${ }^{16}$

\section{Study aims and outcomes}

The primary aim is to report the presentation, management and outcomes of diabetic foot disease within three major centres across Australia and NZ. The secondary 
aims are to determine predictors of wound healing, to determine the predictability of existing wound grading systems and to assess novel tools for measuring lower limb perfusion.

The primary outcomes are the time to wound healing, major amputation, overall mortality and amputation-free survival. The secondary outcomes are the influence of WIfI grading on amputation at 1 year, and the severity of ischaemia in diabetic feet as per PAT.

\section{METHODS AND ANALYSIS \\ Study design}

This is an observational prospective study involving -several major centres (Waikato Hospital, NZ; Sir Charles Gairdner Hospital, the Royal Adelaide Hospital and the Queen Elizabeth Hospital Australia). As an observational study, no intervention will be evaluated. The duration for both participant recruitment and follow-up will be 12 months. Participants will be provided with a Participant Information Sheet and undergo written consent prior to involvement in the study.

\section{Sample selection}

The research population is all patients with diabetes foot disease. The study sample population are consecutive patients presenting with diabetic foot disease, identified from MDT diabetic foot clinic, vascular clinic, dialysis and admission to hospital. The inclusion criteria are those over 18 years old experiencing diabetic foot disease as defined by a diagnosis of diabetes mellitus and with lower limb infection, ulceration, non-healing surgical wounds, gangrene, peripheral neuropathy, lower limb rest pain or foot deformity. ${ }^{1}$ The exclusion criteria are: unwilling to consent/participate, the presence of previous bilateral major limb amputation, persons unable to consent, a documented cognitive impairment condition, persons experiencing a documented palliative medical condition with life expectancy $<12$ months or persons that cannot attend minimum follow-up of 12 months. The withdrawal criterion is patient preference. The pragmatic recruitment approach across multiple services (MDT diabetic foot clinic, vascular clinic, hospital admission and dialysis) at three tertiary sites, enables a large sample size that is representative of the wide range of presentations and disease progression managed in tertiary centres, encompassing low, moderate and high-risk feet, and increases the generalisability of the study.

\section{Recruitment observational data}

At recruitment, the following data will be collected: service details, baseline demographic and clinical history details, wound data on both lower limbs and discharge information (online supplemental appendix 1).

Baseline service details will include the service the participant is attending at the time of recruitment, the date and source of referral to the current service, and all the relevant services/teams that are currently involved with the participant's management.

Baseline demographic and clinical history details will include the participants age in years, gender and the ethnicity that the participant identifies with. The NZ ethnicity selection will align with NZ census and research standards. In Australia, participants will be asked to identify as Aboriginal or Torres Strait Islander, or nonAboriginal/Torres Strait Islander. In addition, the history of diabetes mellitus will be collected including whether the participant has been diagnosed with diabetes, the year of diabetes diagnosis, the type of diabetes the participant is diagnosed with, current glycaemic control with a haemoglobin A1c (HbA1c) dated within the last 3 months. Adjustments will later be made for differing of HbAlc reporting between Australia (\%) and NZ (mol/ $\mathrm{mmol}$ ). Other baseline health status data collection will include recent haemoglobin level and date, presence of transfusion of red blood cells in last 3 months, current dialysis status for renal replacement therapy and dialysis modality, recent estimated glomerular filtration rate (eGFR) and creatinine, smoking history listed as current, ex-smoker less than 12 months, ex-smoker greater than 12 months or non-smoker; and number of smoking pack-years (number of packs of cigarettes smoked per day by the number of years the participant has smoked), concomitant medications that the participant is currently taking. The presence of vascular risk factors will be noted including a family or personal history of ischaemic heart disease (history of angina, myocardial infarction, percutaneous coronary intervention or coronary artery bypass procedure), cerebrovascular accident (history of ischaemic stroke or transient ischaemia attack), peripheral artery disease (signs/symptoms or radiological evidence of atherosclerotic peripheral vascular disease of any peripheral arteries, and ankle brachial pressure index in those with compressible tibial/pedal vessels), hypertension (blood pressure $>130$ systolic or $>80$ diastolic or history of hypertension with presence of antihypertensive medication), hypercholesterolaemia (raised level of total cholesterol and low density lipoprotein, presence of lipid lowering medications, diabetes and smoking history. ${ }^{17}$ In addition, the height $(\mathrm{m})$ and weight $(\mathrm{kg})$ to calculate body mass index, most recent vitamin A, vitamin C, vitamin D, zinc, ferritin and vitamin B12 levels, the average grip strength out of three attempts for both hands using the hydraulic hand dynamometer (Saehan Medical, Gyeongsangnam, South Korea), and the Clinical Frailty Scale (ranging between 1 and 9) produced by Geriatric Medicine Research, Dalhousie University, Canada will be recorded. Also collected will be whether the participant is currently admitted to hospital, recently discharge in the last 1 month with duration of stay or whether admission is required from this review, and any recent vascular or diabetic foot disease-related interventions in the past 6 weeks, including the name of the intervention, the date of angioplasty or lower limb bypass occurred, the details of which vessels were involved in the treatment. 
Baseline wound data will be collected on both lower limbs (including contralateral asymptomatic limbs) including presence of peripheral neuropathy based on a $10 \mathrm{~g}$ monofilament examination according to the IWGDF examination recommendations, ${ }^{18}$ palpable dorsal pedis or posterior tibial pulses, the PAT in milliseconds for both legs via ultrasound examination and date. The foot arteries able to be examined in PAT are the arcuate artery, dorsal metatarsal artery, medial plantar artery, lateral plantar artery and deep plantar artery. The toe pressure measurement will be recorded using Systoe (Atys Medical, Soucieu-en-Jarrest, France) and the Doppler ATP ankle and toe pressure kit (Huntleigh Healthcare Limited, Cardiff, UK). The toe pressure measurement location (great toe or second toe) will be recorded. If the toe pressure is not attainable, the reason for a lack of toe pressure reading, such as multiple toe amputation, forefoot amputation, callus, significant oedema or very poor perfusion will be recorded. The inability to obtain a toe pressure with damped or monophonic waveforms will later be scored in severe ischaemia category ( $\mathrm{i}=3$ ) on WIfI assuming poor perfusion as the cause. A foot deformity score will be calculated for each foot, with one point for, prominent metatarsal heads or bony prominences, hammer or claw toes, small muscle wasting, Charcot, or limited joint ability. ${ }^{19}$ A note will be made of Charcot neuropathy. The reports of imaging in the past 6 months including foot X-ray, arterial lower limb duplex ultrasound, CT, MRI or digital subtraction angiography; and whether surgical revascularisation has occurred since the imaging will be recorded. Evidence of foot deformity on $\mathrm{X}$-rays will be recorded. The current target artery path (TAP) status will be collected. An intact TAP is defined as there being $<50 \%$ stenosis in the iliac and femoral arteries with at least one vessel run off to the foot; and not intact if there is a $\geq 50 \%$ stenosis in the iliac or femoral arteries or there is no vessel run off to the foot. In addition, whether the participant has undergone a minor or major limb amputation in the past, time since amputation and site of the amputation will be recorded; along with whether the participant has a history of ulceration on each foot, the current presence of rest pain, foot ulceration or gangrene. One wound will be chosen on each foot to be the 'index' wound. This will be determined as the highest grade wound on the foot as determined by wound criteria in the WIfI scoring. The description of index wound type being surgical wound, ulcer or gangrene will be collected. A surgical wound is described as a wound created by a surgical procedure such as debridement or amputation, an ulcer being a tissue deficit not surgically created and gangrene as necrosis of tissue. A wound labelled ulcer or gangrene may change to a surgical wound if a surgical debridement or amputation takes place. However, once labelled a surgical wound, the wound shall remain labelled surgical wound until healed. The location, depth and duration in weeks of the index surgical wound, ulcer or gangrene will be collected utilising patient recall and medical records, followed by a description of all foot wounds, ulcer(s) or gangrene and size $\left(\mathrm{mm}^{2}\right)$. If the index wound is a surgical wound, the duration of ulcer or gangrene prior to the first debridement or amputation in weeks will be collected. The depth will be defined as superficial, deep or extensive ulcer based on wound criteria for depth in WIfI scoring. Also recorded will be the highest $\mathrm{C}$ reactive protein (CRP) within the last 2 weeks and date, details of current antimicrobial therapy including antibiotic prescribed and duration of therapy, recent microbiology results relevant to the limb ulcer or wound, sensitivities and date. A wound, ischaemia, and foot infection grade based on WIfI for each limb will be recorded (online supplemental appendix 2) and used to calculate the WIfI score for amputation risk at 1 year and benefit of revascularisation. The current offloading measures are defined as no offloading, removable or irremovable ankle (or knee) high device, therapeutic footwear and surgical offloading.

Baseline discharge information will record the service discharge destination including diabetic foot clinic, general practitioner care, acute hospital admission, waitlist for surgery, palliative care, residential aged care service or patient discharged self at risk.

\section{Review observational data}

Participants will be reviewed at 1, 3, 6 and 12 months during the 1 year follow-up occurring at subsequent clinic appointments or hospital admissions (online supplemental appendix 3). Unless italicised, data collected in the review consultation has the same definition as the recruitment information.

Review service details will include the service attended, and all the relevant services/teams currently involved in management.

Review demographic and clinical history details will include diabetes diagnosis, HbAlc dated within the last 3 months, recent haemoglobin level and date, blood transfusion in last 3 months, grip strength and the Clinical Frailty Scale (ranging between 1 and 9 ). Also, whether the participant is currently admitted to hospital, recent discharge since last review with duration of stay or admission required from this review; and any recent vascular or diabetic foot disease-related interventions since last review.

Review wound data will be presence of palpable pedal pulses, recent PAT and date, toe pressure, toe pressure location and reason for a lack of toe pressure reading. Recent imaging reports since last review, whether surgical revascularisation has occurred since the imaging and the TAP status. The current presence of rest pain, foot ulceration or gangrene will be collected. The index wound type, location, depth and duration will be recorded, followed by a description of all foot wounds, ulcer(s) or gangrene and size $\left(\mathrm{mm}^{2}\right)$. If the index is now a surgical wound, the duration of ulcer or gangrene prior to the first debridement or amputation in weeks will be collected. The highest CRP within the last 2 weeks and date, details of current antimicrobial therapy, recent microbiology results relevant to the limb ulcer or wound, sensitivities and date. The WIfI 
grades and WIfI score for amputation risk at 1 year and benefit of revascularisation will be recorded. The current offloading measures will be recorded.

Review discharge information will record the service discharge destination and the outcomes of whether the right or left foot index wound has healed defined as complete re-epithelisation of the previous tissue defect and date of healing; whether a right or left major limb amputation has occurred defined as the removal of a limb above the ankle and date; whether the participant has died and date of death.

\section{Sample size}

Based on annual volume numbers, this study will be able to include 150-200 patients at three centres, completing a total of 450-600 participants.

\section{Data analysis plan}

The primary and secondary outcomes will be analysed as follows. Continuous variables will be presented as mean and SD for parametric data, and median and IQR for non-parametric data. Clinically important covariates such as age, sex, WIfI stage, renal disease, HbAlc levels will be selected according to a priori from the published literature to include in all adjusted regression models.

For binary outcomes (yes/no) such as amputation free survival, major amputation and wound healing, clinically known variables that may have an impact on 30-day and 1 year death will be entered into a logistic regression model and analysed at a univariate level. Covariates with a $p$ value $<0.2$ will then be added into a multivariate logistic regression model and the impact of predictors will be expressed as OR and 95\% CIs.

For time to event (death, amputation and wound healing) analysis, Kaplan-Meier curves will be performed at univariate levels to determine variables that might influence the outcome of interest. Covariates with a $p$ value $<0.2$ will be entered into a Cox proportional hazard models to estimate HR and $95 \%$ CI.

In addition, exploratory variables such as sex-adjusted grip strength, Clinical Frailty Scale, vitamin levels will also be tested in model development. For all multi-regression models developed, the 1 in 10 rule will be adopted and all efforts will be made to prevent any violation and overfitting.

\section{Patient and public involvement}

No patient involved.

\section{ETHICS AND DISSEMINATION \\ Ethical and safety considerations}

This study has received the following approvals: New Zealand Central Health and Disability Ethics Committee (20/CEN/122), Waikato DHB Research Department (RDO020044), Quality Improvement HoD Sir Charles Gairdner Hospital (39715), and the Central Adelaide
Local Health Network (CALHN) Human Research Ethics Committee (13928).

This is an observational study hence any clinical management decisions are made by the participant's treating team.

To share deidentified data between the Australian and NZ sites, this study will use Research Electronic Data Capture (REDCap, Vanderbilt University, USA). This is a secure, web-based application supporting data capture for research studies. Access to REDCap will be via the licence held by The University of Auckland, and only approved researchers from other sites will be granted access this database to add deidentified data. Health information captured in REDCap will be stored on a secure server at the University of Auckland. REDCap requires a two-factor authentication and once logged on, a user is only able to view a project they created or a project they have been invited to.

\section{Dissemination plan}

The analysed results will be presented at international conferences as deemed relevant for vascular surgery, diabetic foot disease and podiatry. In addition, the results will be prepared for publication in peer-reviewed journals.

Results or publications will not be individually provided to participants, however a summary of the outcomes will be provided to participants if they indicate their wish to receive this at the consent consultation. Raw data will not be made publicly available to uphold the confidentiality and privacy of participants given the prospective nature of observational data collection.

For publication policy, there will be prior agreed on authorship listing for each article produced from data collected in this study. Hence, a lead will be assigned for each publication, with subsequent authors to include site investigators, supervisors and collaborators as appropriate. All authors listed will have met the requirements as having been involved in the concept, design, implementation and review of any publications (as defined by the ICJME).

\section{Data storage}

Health information collected on hard copy records will be secured in a locked cabinet assessable only by the lead researcher at the respective centre. A password-protected master file will be kept at each centre which links the participant to their unique identifier in REDCap. This master file will be stored on secure servers with firewalls at institutional network drives. All health information will be kept for a minimum period of 10 years in $\mathrm{NZ}$ and a minimum of 15 years in Australia. After this time, all data will be destroyed by shredding if in paper form or permanently deleted from all computer storage.

\section{Author affiliations}

${ }^{1}$ Department of Surgery, Faculty of Medical and Health Sciences, The University of Auckland, Auckland, New Zealand

${ }^{2}$ Department of Vascular Surgery, Waikato Hospital, Hamilton, New Zealand

${ }^{3}$ Curtain Medical School, Curtin University, Perth, Western Australia, Australia 
${ }^{4}$ Surgery Division, Faculty of Health and Medical Sciences, The University of Western Australia, Perth, Western Australia, Australia

${ }^{5}$ Department of Vascular and Endovascular Surgery, Sir Charles Gairdner Hospital, Perth, Western Australia, Australia

${ }^{6}$ Discipline of Surgery, The University of Adelaide, Adelaide, South Australia, Australia

${ }^{7}$ Department of Vascular Surgery, Royal Adelaide and Queen Elizabeth Hospitals, Adelaide, South Australia, Australia

Acknowledgements We would like to acknowledge Tass Borman, Claire 0'Shea, Prue Cowled, Neil McMillan, Claire Murray and Jill Sommerset for their contributions.

Contributors $\mathrm{OH}$ contributed to protocol conception and design, development of statistical analysis, data collection, writing the protocol manuscript, critical revision and final approval of the protocol manuscript, and obtaining funding. SJ contributed to protocol conception and design, critical revision and final approval of the protocol manuscript, and obtaining funding. RAF contributed to protocol conception and design, critical revision and final approval of the protocol manuscript, and obtaining funding. MK contributed to protocol conception and design, development of statistical analysis, critical revision and final approval of the protocol manuscript, and obtaining funding.

Funding This work was supported by the 2020 ANZSVS Vascular Foundation Project Grant and the Vascular and Radiology Research Fund (Western Australia Health Department).

Competing interests None declared.

Patient consent for publication Not required.

Provenance and peer review Not commissioned; externally peer reviewed.

Supplemental material This content has been supplied by the author(s). It has not been vetted by BMJ Publishing Group Limited (BMJ) and may not have been peer-reviewed. Any opinions or recommendations discussed are solely those of the author(s) and are not endorsed by BMJ. BMJ disclaims all liability and responsibility arising from any reliance placed on the content. Where the content includes any translated material, BMJ does not warrant the accuracy and reliability of the translations (including but not limited to local regulations, clinical guidelines, terminology, drug names and drug dosages), and is not responsible for any error and/or omissions arising from translation and adaptation or otherwise.

Open access This is an open access article distributed in accordance with the Creative Commons Attribution Non Commercial (CC BY-NC 4.0) license, which permits others to distribute, remix, adapt, build upon this work non-commercially, and license their derivative works on different terms, provided the original work is properly cited, appropriate credit is given, any changes made indicated, and the use is non-commercial. See: http://creativecommons.org/licenses/by-nc/4.0/.

\section{ORCID iDs}

Odette Hart http://orcid.org/0000-0002-6330-2810

Shirley Jansen http://orcid.org/0000-0001-7781-4748

Robert Fitridge http://orcid.org/0000-0001-6258-5997

Manar Khashram http://orcid.org/0000-0003-4921-8433

\section{REFERENCES}

1 van Netten JJ, Bus SA, Apelqvist J, International Working Group on the Diabetic Foot guidelines. Definitions and criteria for diabetic foot disease. Diabetes Metab Res Rev 2019;36.

2 Cavanagh PR, Lipsky BA, Bradbury AW, et al. Treatment for diabetic foot ulcers. The Lancet 2005;366:1725-35.

3 Armstrong DG, Boulton AJM, Bus SA. Diabetic foot ulcers and their recurrence. N Engl J Med Overseas Ed 2017;376:2367-75.

4 Gurney JK, Stanley J, York S, et al. Risk of lower limb amputation in a national prevalent cohort of patients with diabetes. Diabetologia 2018;61:626-35.

5 Boulton AJM, Vileikyte L, Ragnarson-Tennvall G, et al. The global burden of diabetic foot disease. Lancet 2005;366:1719-24.

6 Lazzarini PA, van Netten JJ, Fitridge RA, et al. Pathway to ending avoidable diabetes-related amputations in Australia. Med $\mathrm{J}$ Aust 2018;209:288-90.

7 Joret MO, Dean A, Cao C, et al. The financial burden of surgical and endovascular treatment of diabetic foot wounds. J Vasc Surg 2016:64:648-55.

8 West M, Chuter V, Munteanu S, et al. Defining the gap: a systematic review of the difference in rates of diabetes-related foot complications in Aboriginal and Torres Strait Islander Australians and non-Indigenous Australians. J Foot Ankle Res 2017;10:48.

9 Robinson TE, Kenealy T, Garrett M, et al. Ethnicity and risk of lower limb amputation in people with type 2 diabetes: a prospective cohort study. Diabet Med 2016;33:55-61.

10 Monteiro-Soares M, Russell D, Boyko EJ. International Working group on the diabetic foot guideline: on the classification of diabetic foot ulcers, 2019

11 Mills JL, Conte MS, Armstrong DG, et al. The Society for vascular surgery lower extremity threatened limb classification system: risk stratification based on wound, ischemia, and foot infection (WlfI). $J$ Vasc Surg 2014;59:e1-2:220-34.

12 Conte MS, Bradbury AW, Kolh P, et al. Global vascular guidelines on the management of chronic limb-threatening ischemia. Eur $J$ Vasc Endovasc Surg 2019;58:e33:S1-109.

13 Forsythe RO, Hinchliffe RJ. Assessment of foot perfusion in patients with a diabetic foot ulcer. Diabetes Metab Res Rev 2016;32 Suppl 1:232-8.

14 Sommerset J, Karmy-Jones R, Dally M, et al. Plantar acceleration time: a novel technique to evaluate arterial flow to the foot. Ann Vasc Surg 2019;60:308-14.

15 Health Research Council of New Zealand. The New Zealand health research prioritisation framework. Wellington: Ministry of Business, Innovation and Employment and the Ministry of Health, 2019.

16 Hinchliffe RJ, Forsythe RO, Apelqvist J, et al. Guidelines on diagnosis, prognosis, and management of peripheral artery disease in patients with foot ulcers and diabetes (IWGDF 2019 update). Diabetes Metab Res Rev 2020;36 Suppl 1:e3276.

17 Ministry of Health. Cardiovascular disease risk assessment and management for primary care. Wellington: Ministry of Health, 2018.

18 Schaper NC, van Netten JJ, Apelqvist J. IWGDF practical guidelines on the prevention and management of diabetic foot disease, 2019.

19 Abbott CA, Chatwin KE, Foden P, et al. Innovative intelligent insole system reduces diabetic foot ulcer recurrence at plantar sites: a prospective, randomised, proof-of-concept study. Lancet Digit Health 2019;1:e308-18. 